\title{
ESPECIES NEOTROPICALES DE LECANIOBIUS ASHMEAD (HYMENOPTERA: CHALCIDOIDEA: EUPELMIDAE): CLAVE Y DESCRIPCIÓN DE DOS ESPECIES NUEVAS
}

\author{
Svetlana N. Myartseva, Enrique Ruíz-CanCino \\ y Juana María CORONADO-Blanco
}

División de Estudios de Postgrado e Investigación, UAM Agronomía y Ciencias, Universidad

Autónoma de Tamaulipas, 87149 Ciudad Victoria, Tamaulipas, MÉXICO.

E-mail: smyartse@uat.edu.mx; eruiz@uat.edu.mx; jmcoronado@uat.edu.mx

Myartseva S. N., E. Ruíz-Cancino \& J. M. Coronado-Blanco. 2010. Especies neotropicales de Lecaniobius Ashmead (Hymenoptera: Chalcidoidea: Eupelmidae): clave y descripción de dos especies nuevas. Acta Zool. Mex. (n. s.), 26(3): 669-683.

RESUMEN. Se estudiaron las especies neotropicales del género Lecaniobius y se elaboró la clave para su identificación. Se describieron dos especies, Lecaniobius mexicanus n. sp. de México y $L$. nicaraguensis n. sp. de Nicaragua, y se redescribieron L. capitatus Gahan y L. cockerellii Ashmead, incluyendo algunas características morfológicas adicionales.

Palabras clave: Hymenoptera, Eupelmidae, Lecaniobius, México.

Myartseva S. N., E. Ruíz-Cancino \& J. M. Coronado-Blanco. 2010. Neotropical species of Lecaniobius Ashmead (Hymenoptera: Chalcidoidea: Eupelmidae): key and description of two new species. Acta Zool. Mex. (n. s.), 26(3): 669-683.

ABSTRACT. A review of neotropical species of the genus Lecaniobius is given, with a key to identify it. Lecaniobius mexicanus sp. n. from Mexico and L. nicaraguensis sp. n. from Nicaragua are described; L. capitatus Gahan and L. cockerellii Ashmead are redescribed, including some additional morphological characteristics.

Key words: Hymenoptera, Eupelmidae, Lecaniobius, Mexico.

\section{INTRODUCCIÓN}

El género Lecaniobius Ashmead, 1896 pertenece a la familia de avispas parasíticas Eupelmidae. Se conocen más de 850 especies y 45 géneros de esta familia a nivel mundial (Gibson 1995a). Eupelmidae está dividida en tres subfamilias, incluyendo Eupelminae. Los géneros de Eupelminae fueron revisados por Gibson (1995a). Se conocen 33 géneros en el mundo, de los cuales 25 se encuentran en la región neotropical y 14 en la neártica. Todos los géneros neárticos se hallan también en la región neotropical y la mitad sólo se reportan del sur de Estados Unidos (EU). Su

Recibido: 16/02/2010; aceptado: 04/06/2010. 
diversidad genérica y específica parece ser resultado de la distribución de géneros centrados en los neotrópicos.

Lecaniobius fue descrito en 1896 por Ashmead con material del Nuevo Mundo pero hasta el presente ha sido muy poco estudiado. Las especies fueron revisadas por Compere (1939). El género incluye cuatro especies distribuídas en la región neotropical y en la parte sur de la neártica: L. capitatus Gahan, L. cockerellii Ashmead, L. grandis De Santis y L. utilis Compere (De Santis 1979). Las especies de Lecaniobius son depredadoras de huevos o parasitoides secundarios en escamas (Smith y Compere 1928), especialmente de la familia Coccidae (p. ej., Saissetia). Dos especies fueron introducidas al sur de EU para el control biológico de escamas lecaniinas (Coccidae).

Para México sólo se había registrado una especie, L. capitatus (GonzálezHernández 2000, Ruíz-Cancino y Coronado-Blanco 2002), la cual fue introducida para el control biológico de la escama Saissetia oleae (Olivier) y estaba registrada de los estados de Colima, D.F., Guanajuato, Morelos, Nuevo León y Tamaulipas (Myartseva et al. 2004).

El presente trabajo contiene la descripción de dos especies nuevas y la redescripción de otras dos especies presentes en México, además se consideran algunas características morfológicas adicionales y se elaboró una clave para la identificación de las seis especies neotropicales de Lecaniobius.

\section{MATERIALES Y MÉTODOS}

Durante los últimos años se colectaron hojas de varias plantas infestadas con escamas suaves del género Saissetia (Hemiptera: Coccidae) en los estados de Tamaulipas y Tabasco, México. Este material se trasladó al Laboratorio de Control Biológico de la UAM Agronomía y Ciencias-UAT, donde se colocó en viales pequeños de vidrio para detectar el parasitismo. De dicho material se obtuvieron varios parasitoides pertenecientes al género Lecaniobius Ashmead, incluso una especie nueva. En adición, en una estancia académica efectuada en diciembre de 2009 en el Museo Essig de la Universidad de California en Berkeley, EU, se encontró una nueva especie de Eupelmidae de Nicaragua del mismo género.

Se revisaron las descripciones originales de las cuatro especies conocidas del género, además de los paratipos de L. cockerellii Ashmead depositados en el Museo Essig. Para estudiar las estructuras morfológicas, una parte de los especímenes se pegaron en triángulos de cartulina por el lado derecho del tórax y otros especímenes fueron disectados y montados en laminillas con bálsamo de Canadá, según lo propuesto por Noyes (1982). Todos los especímenes fueron etiquetados.

En la redescripción de las especies no se consideró la escultura y la pubescencia ya que estas características fueron registradas a detalle en las descripciones originales. Los especímenes mexicanos fueron depositados en el Museo de Insectos 
de la Universidad Autónoma de Tamaulipas, en Cd. Victoria, Tamaulipas, México. Los acrónimos de los depositantes de los tipos son: UCRC - Universidad de California, Riverside, California, EU; USNM - U.S. National Museum of Natural History, Washington D.C., EU.

\section{RESULTADOS Y DISCUSIÓN}

Los especímenes mexicanos fueron identificados como L. capitatus Gahan y $L$. cockerellii Ashmead. Por otra parte, se describieron dos especies nuevas y se redescribieron dos conocidas, lo que permitió preparar la revisión de las seis especies de este género neotropical y elaborar la clave para la identificación de especies de Lecaniobius, la cual se presenta a continuación.

\section{Clave de especies del género Lecaniobius (hembras)}

1. Margen dorsal del basitarso de la pata posterior blanco. Funículo antenal completamente parduzco ............................. nicaraguensis Myartseva, n. sp.

- Basitarso de la pata posterior de pardo a negro. Color del funículo antenal variable ....2

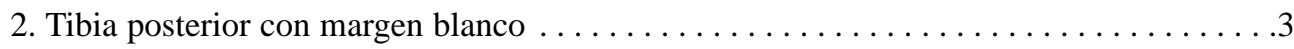

- Tibia posterior completamente de parda a negra $\ldots \ldots \ldots \ldots \ldots \ldots \ldots \ldots . \ldots . \ldots . \ldots$

3.Cabeza, incluyendo el occipucio, completamente pardo claro ....L. cockerellii Ashmead - Cabeza completamente negra, con brillo metálico ............... grandis De Santis

4. Cabeza completamente negra, con brillo metálico. Setas normales en la región entre las escrobas antenales (no aplanadas) ................. mexicanus Myartseva, n. sp.

- Cabeza con patrones pardo claro. Setas aplanadas y agrandadas en la región entre las

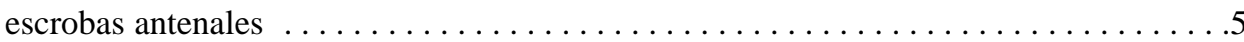

5. Ala anterior con la banda obscura ancha distal sin borde contrastante de cilios blancos; base del ala generalmente ciliada, excepto a lo largo del margen posterior ....................................... utilis Compere

- Ala anterior con la banda obscura ancha distal marginada por un borde angosto contrastante de cilios blancos; base del ala con un parche oblicuo de cilios oscuros

.L. capitatus Gahan 
Myartseva et al.: Especies neotropicales de Lecaniobius Ashmead

\section{Revisión de las especies \\ 1. Lecaniobius capitatus GAHAN}

(Figs. 1-5)

Lecaniobius capitatus Gahan 1924: 4-5. Holotipo ㅇ, Las Sabanas, Panamá. USNM.

\section{Descripción. Hembra.}

Coloración. Cabeza pardo claro, frontovértice así como las carinas a los lados de los surcos antenales, área interantenal y mejillas de pardo a negro con brillo metálico. Pubescencia de la cabeza de color blanco plateado. Palpos labiales y maxilares amarillo claro, con los 2 segmentos apicales negro parduzco. Antenas de pardo a negro, escapo y segmentos apicales funiculares pardo claro. Pronoto, mesoescudo y lados del tórax de pardo a negro con brillo metálico, axilas negras con brillo metálico, escutelo de amarillo oscuro a pardo claro. Patas frontales de pardo a negro, tarsos ligeramente más claros, coxas con brillo metálico verde. Patas medias de parduzcas a negras, coxas y fémures basalmente pardo claro, fémures con una mancha blanca en el ángulo apical anterior, espuela amarillo blancuzco, segmentos tarsales III y IV a veces más claros. Patas posteriores de color pardo a negro, coxas y fémures pardo claro en el lado interior. Alas anteriores con una banda transversal negra ancha medialmente, la cual está marginada proximal y distalmente por un borde contrastante angosto de cilios blancos; base con un área elongada de cilios blancos que se extienden oblicuamente desde la base de la vena submarginal; tercio apical del ala hialino-parduzco. Venas infuscadas excepto la parte apical de la vena submarginal. Alas posteriores completamente hialinas. Gáster negro con brillo metálico, base parduzca ventralmente. Vainas del ovipositor expuestas, de amarillo a parduzco, bordeadas con negro.

Estructura. Cabeza más ancha que el tórax en vista dorsal, cerca de 1.3 veces tan ancha como alta; frontovértice cerca de 0.5 veces la anchura de la cabeza (Fig. 1). El área entre el margen interior del ojo y el margen escrobal es casi tan ancha como en el margen inferior de los ojos. Ocelos en ángulo obtuso; ocelos posteriores separados de los márgenes oculares por una distancia de 1.5-2.0 diámetros de un ocelo. Ojos cerca de 1.8 veces tan largos como las mejillas. Suturas genales completas. Palpos labiales y maxilares de cuatro y tres segmentos, respectivamente; mandíbula tridentada (Figs. 11-13). Antenas insertadas inmediatamente en el nivel del margen inferior de los ojos (Fig. 2). Distancia entre tórulos 1.5 veces tan larga como la distancia al margen bucal y cerca de 3.0 veces tan larga como la distancia al margen ocular. Cara y frontovértice con pubescencia corta, densa y blanca, región interantenal con setas cortas, lanceoladas, en forma de escamas. Ojos desnudos. Escapo antenal cilíndrico, cerca de 6.5 veces tan largo como ancho; pedicelo aproximadamente 2.0 veces tan largo como ancho; anelo ligeramente más ancho que largo; primer segmento funicular 2.0 veces tan largo 
como ancho, segundo y tercer segmentos ligeramente más cortos que el primero, quinto segmento ligeramente más largo que ancho, subcuadrado; sexto y séptimo segmentos cerca de 1.5 veces tan anchos como largos; maza oblicuamente truncada, tan larga como los tres segmentos funiculares precedentes juntos. Segmentos funiculares II-VII y segmentos de la maza antenal con sensilas longitudinales. Escutelo con hilera media de setas robustas. Ala anterior cerca de 2.5 veces tan larga como ancha; vena marginal muy ligeramente más corta que la vena submarginal; vena postmarginal y estigmal subiguales en longitud. Fémures frontales abultados distalmente, tibias ensanchadas (Fig. 3). Basitarso de las patas medias (Fig. 4) ligeramente expandido, más o menos tan largo como los siguientes tres segmentos tarsales siguientes juntos, segmentos tarsales 1-3 con dos hileras de espinas cortas en el lado inferior. Tibias posteriores (Fig. 5) algo comprimidas y ligeramente más largas que los fémures. Gáster ligeramente más corto que el tórax; ovipositor expuesto.

Longitud del cuerpo: 2.4-3.0 mm.

Macho. Desconocido.

Comentarios. Lecaniobius capitatus es cercana a L. cockerellii, se distingue por las siguientes características: en $L$. cockerellii - cabeza completamente parduzca y 1.5 veces tan ancha como larga, área entre el margen interior del ojo y el margen escrobal es más angosta en el margen inferior de los ojos, escapo antenal cerca de 5.0 veces tan largo como ancho o más corto, región interantenal con pubescencia corta; ovipositor no expuesto; en L. capitatus - cabeza con patrones negros y 1.3 veces tan ancha como alta, área entre el margen interior del ojo y el margen escrobal es casi tan ancha como en el margen inferior de los ojos, escapo antenal cerca de 6.5 veces tan largo como ancho, región interantenal con setas lanceoladas parecidas a escamas; ovipositor expuesto.

Distribución. Panamá, Uruguay, EU (Texas) (Burks 1979, Noyes 2003); México - Colima, D.F., Morelos, Nuevo León (González-Hernández 2000); Tamaulipas es un nuevo registro de distribución.

Huéspedes. Ceroplastes sp., Parasaissetia nigra (Nietner), Saissetia coffeae (Walker), S. oleae (Olivier).

Material examinado. México: Tamaulipas, Tampico, 4우, ex Saissetia sp. en árbol desconocido, 27.III.2001; 19, ex Saissetia sp. en Psidium guajava (col. S.N. Myartseva); Ciudad Victoria, Lienzo Charro, en follaje con fruto de Cedrela odorata, 1+, 31.VIII.2000 (col. E. Ruíz-Cancino). 


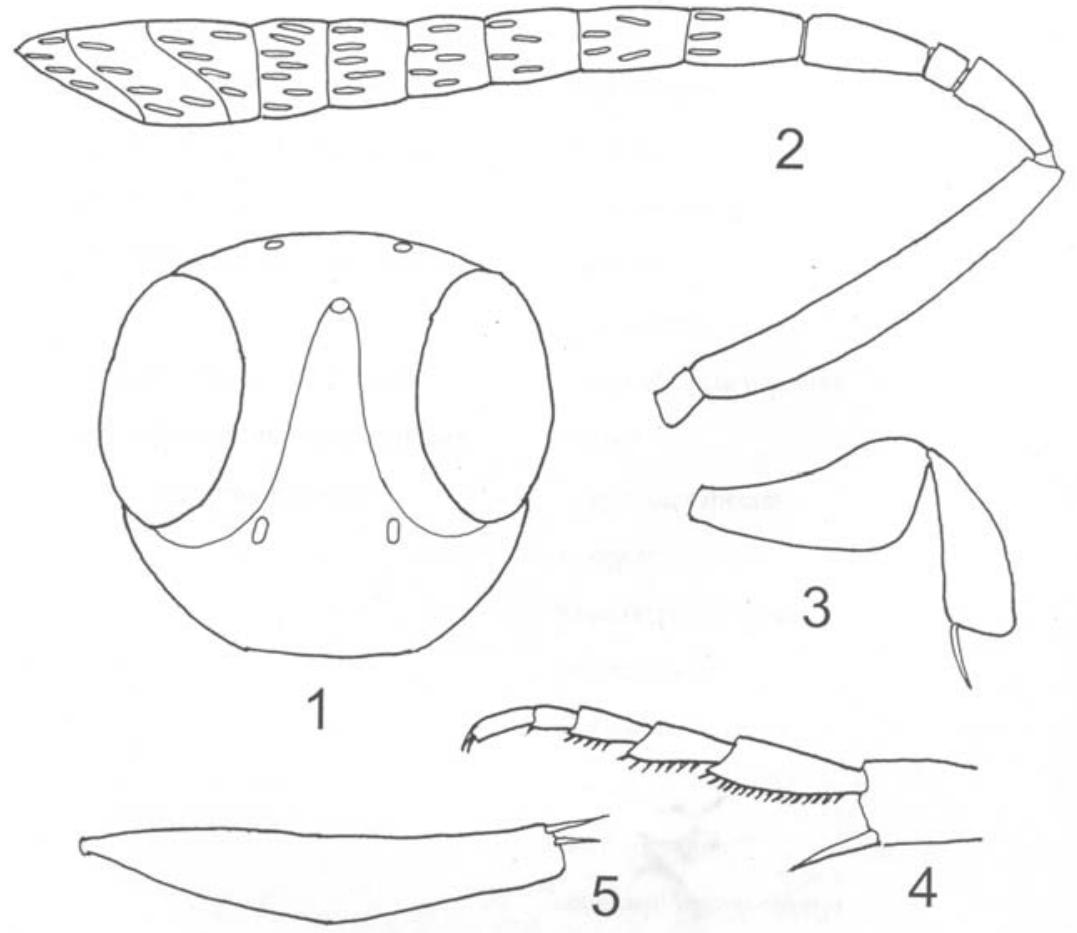

Figuras. 1-5. Lecaniobius capitatus Gahan, hembra: 1 - cabeza, 2 - antena, 3 - fémur y tibia frontal, 4 - espuela tibial y tarso medio, 5 - tibia posterior.

\section{Lecaniobius cockerellii Ashmead}

(Figs. 6-10)

Lecaniobius cockerellii Ashmead, 1896: 17-18. Holotipo 9 , Antigua, British West Indies. USNM.

Zalophothrix mirum Crawford 1908: 156-157. Sinonimia por Crawford 1911: 275. Descripción. Hembra.

Coloración. Cabeza parduzca, frontovértice pardo oscuro y con brillo metálico. Escapo antenal parduzco, flagelo negro parduzco. Palpos labiales y maxilares amarillo obscuro. Pronoto y mesoescudo pardo oscuro y con brillo metálico. Escutelo parduzco, setas negras en la hilera media. Alas anteriores con una banda oscura ancha que se extiende desde la vena marginal y la estigmal hasta el borde posterior del ala, y con un área oblicua oscura debajo de la base de la vena submarginal; venas marginal, postmarginal y estigmal infuscadas. Alas posteriores hialinas. Patas de pardo oscuro a negro; tarsos medios amarillos con el último segmento infuscado, 
cerdas negras en el lado inferior de los segmentos tarsales I-III; tibias posteriores con margen dorsal blanco. Gáster de pardo a negro con brillo metálico.

Estructura. Cabeza considerablemente más ancha que el mesosoma y cerca de 1.5 veces más ancha que alta (Fig. 6). Frontovértice cerca de 0.5 veces la anchura de la cabeza y más o menos tan ancha como larga. Ocelos en un pequeño ángulo obtuso; distancia entre el ocelo posterior y el margen ocular ligeramente más corta que la distancia entre los ocelos posteriores. Suturas genales completas. Mandíbula tridentada; palpos maxilares y labiales de cuatro y tres segmentos, respectivamente (Figs. 11-13). Ojos cerca de 1.4-1.5 veces tan largos como las mejillas. Área entre el margen interior del ojo y el margen escrobal más angosta en el nivel inferior de los ojos. Antenas insertadas inmediatamente arriba del nivel del margen inferior de los ojos (Fig. 7). Distancia entre tórulos casi tan larga como la distancia entre un tórulo al margen bucal y cerca de 2.0 veces más corta que la distancia al margen ocular. Radícula muy corta, escapo cilíndrico, ligeramente curvado, cerca de 5 veces tan largo como ancho (en las laminillas 4.4 veces tan largo como ancho). Pedicelo 1.6-1.7 veces tan largo como ancho. Anelo 2.0 veces tan ancho como largo. Segmentos funiculares I y II cerca de 1.7 veces tan largos como anchos, segmento III subcuadrado, segmentos IV-VII ligeramente más anchos que largos. Maza truncada oblicuamente, más o menos tan larga como los 3 segmentos funiculares precedentes juntos. Segmentos funiculares IIIVII y segmentos de la maza con sensilas longitudinales. Escutelo con una hilera media de cerdas. Ala anterior 2.6-3.0 veces tan larga como ancha; vena marginal visiblemente más corta que la vena submarginal. Venas postmarginal y estigmal subiguales en longitud. Fémur frontal abultado distalmente (Fig. 9). Basitarso de las patas medias casi no expandido (Fig. 8), más o menos tan largo como los segmentos tarsales 2-4 juntos; segmentos tarsales 1-3 con dos hileras de espinas cortas en el lado inferior. Tibia posterior algo comprimida y ligeramente más larga que el fémur (Fig. 10). Gáster ligeramente más corto que el tórax. Ovipositor no expuesto; tercera válvula 0.3 veces tan larga como el segundo valvifer.

Longitud del cuerpo: $2.5 \mathrm{~mm}$.

Macho. Desconocido.

Comentarios. Las diferencias de L. cockerellii con las otras especies del género se anotaron en los comentarios de L. capitatus y en la clave de especies. La especie tropical y subtropical Lecaniobius cockerellii es depredadora de huevos de la escama negra Saissetia oleae pero puede convertirse en parasitoide secundario cuando se presenta la oportunidad (Smith \& Compere 1928). Esta especie fue introducida varias veces a California contra la escama negra pero sin éxito (Smith 1923, Smith \& Compere 1928, Essig 1931, Flanders 1953, Bartlett 1978). No se conocía de su existencia en California (Smith 1944). L. cockerellii es un nuevo registro para México, donde fue obtenido en el estado de Tabasco de Parasaissetia nigra en Ficus sp. 


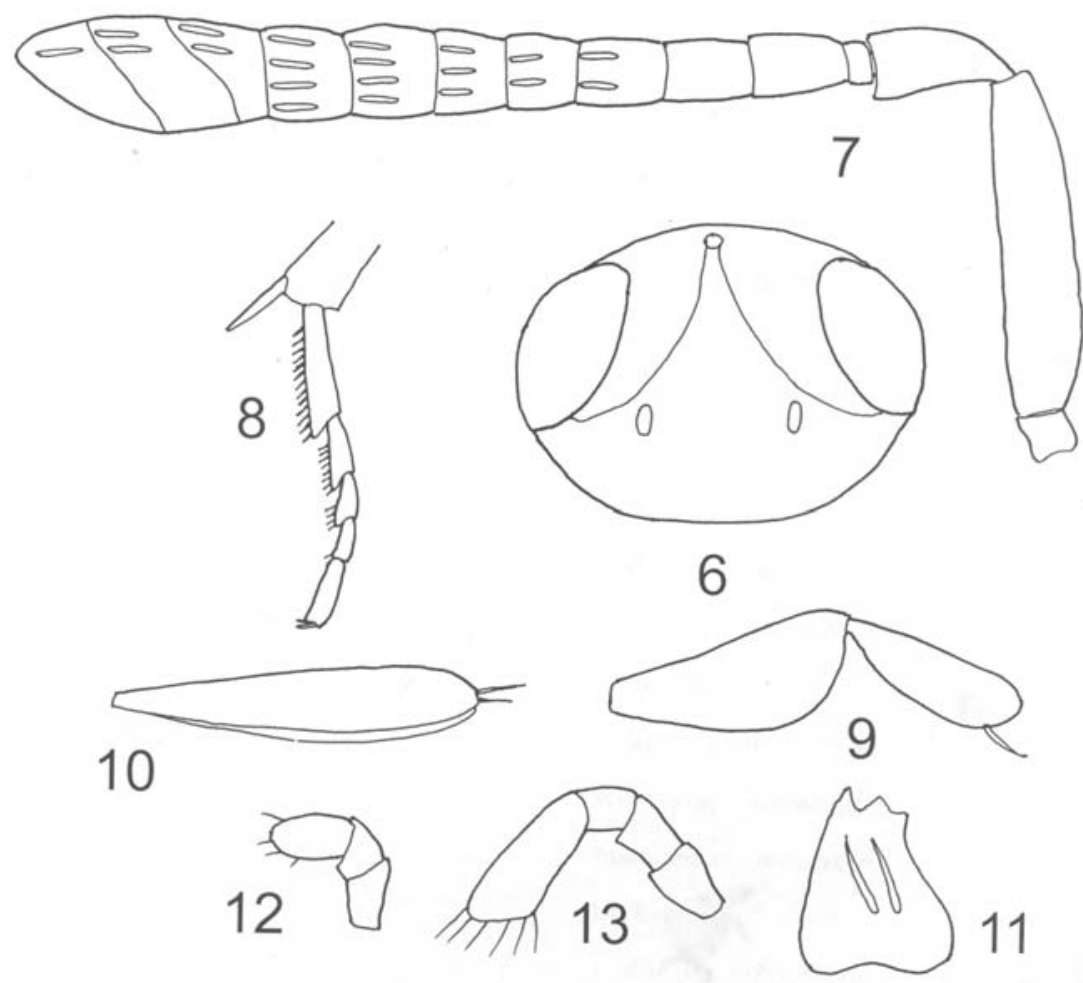

Figuras 6-13. Lecaniobius cockerellii, hembra: 6 - cabeza, 7 - antena, 8 - espuela tibial y tarso medio, 9 - fémur y tibia frontal, 10 - tibia posterior. 11-13. L. grandis De Santis (tomada de De Santis 1950): 11 - mandíbula, 12 - palpo labial, 13 - palpo maxilar.

Distribución. Centro y Sudamérica, EU (California); México (Tabasco) es un nuevo registro de distribución.

Huéspedes. Coccus hesperidum (Linnaeus), C. longulus (Douglas), Parasaissetia nigra (Nietner), Saissetia coffeae (Walker), S. oleae (Olivier); Scutellista cyanea Motschulsky.

Material examinado. EU, California, Colección Entomológica de la California Academy of Sciences: Bermuda, 2ㅇ, Sept. 1921, ex Hibiscus scale, Seabright, O. Degenter coll. México: Tabasco, Villahermosa, 5ㅇ, 10, ex Parasaissetia nigra en Ficus sp., 6.XI.2009 (col. S.N. Myartseva). 


\section{Lecaniobius grandis De Santis}

(Figs. 11-13)

Lecaniobius grandis De Santis, 1950: 1-5. Sintipo 9 , Buenos Aires, Argentina. Museo de La Plata, Argentina.

Cordyloodera incognita Blanchard 1956: 160-164.

Cordyloodera incognita Griot, 1955:176 (nomen nudum). Sinonimia por De Santis et al. 1986: 208.

\section{Descripción. Hembra.}

Coloración. Cabeza de parda a negra con brillo verde azulado metálico. Antenas negruzcas con escapo pardo claro. Palpos labiales y maxilares negro parduzco. Tórax negro parduzco, escutelo parduzco. Axilas con brillo verde. Gáster negro con brillo metálico, el tercio basal parduzco. Patas pardo oscuro, tarsos amarillos en las patas medias, tarsos posteriores parduzcos. Tibia media y espuela pardo claro, tibia posterior con margen dorsal blanco. Alas anteriores con una banda transversal ancha oscura medialmente, bordeada desde la parte apical del ala por una banda hialina angosta, la cual no llega al margen posterior del ala. Venas marginal, postmarginal y estigmal infuscadas. Gáster de pardo a negro, base ventralmente parduzca.

Estructura. Cabeza ligeramente más ancha que alta. Ojos desnudos. Suturas genales completas. Mándíbulas, palpos labiales y maxilares como en las Figs. 11-13. Antenas insertadas al nivel del margen inferior de los ojos. Escapo 6-7 veces tan largo como ancho (en la figura de Blanchard, 1956 mide 5.4x); pedicelo 1.8-2.0 veces tan largo como ancho; anelo 1.5 veces tan ancho como largo; segmentos funiculares antenales ligeramente acortados hacia el ápice, segmento I es 2.0-2.3 veces tan largo como ancho, segmentos IV y V subcuadrados, segmentos VI y VII ligeramente más anchos que largos. Maza antenal más o menos tan larga como los dos segmentos funiculares precedentes juntos (en la figura de Blanchard 1956). Todos los segmentos flagelares con sensilas longitudinales. Alas anteriores 2.1-2.5 veces tan largas como anchas; vena marginal visiblemente más corta que la vena submarginal; venas postmarginal y estigmal subiguales en longitud. Gáster más o menos tan largo como el tórax. Ovipositor expuesto.

Longitud del cuerpo: 4.0-4.2 mm.

Macho. Desconocido.

Distribución. Argentina, Brasil, Uruguay (Noyes 2003).

Huésped. Ceroplastes grandis Hempel.

Comentarios. Lecaniobius grandis es una especie cercana a L. capitatus pero difiere en los siguientes caracteres: L. capitatus - cabeza parduzca debajo de los tórulos, fémures medios con una mancha blanca en el ápice, tarsos medios pardos, tibias posteriores pardas, longitud del cuerpo 2.4-3.0 mm; L. grandis - cabeza de parda a negra con brillo metálico verde azulado, fémures medios sin mancha blanca en el ápice, tarsos medios amarillos, tibias posteriores con el margen dorsal blanco. 
4. Lecaniobius mexicanus Myartseva, n. sp.

Holotipo. ㅇ, México: Tamaulipas, ex Saissetia sp. en árbol desconocido,

27.III.2001 (col. S.N. Myartseva); depositado en UCRC.

\section{Descripción. Hembra.}

Coloración. Cabeza negra con brillo metálico verde azulado bronceado. Antenas negras. Tórax negro con brillo metálico verde azulado bronceado. Axilas de color verde metálico. Alas anteriores hialinas, con una banda infuscada más o menos angosta que se extiende desde la parte apical de la vena submarginal hasta el borde posterior del ala. Parte apical de las venas submarginal, marginal y postmarginal así como el estigma infuscados. Patas anteriores pardas, coxas negras, tarsos amarillos. Patas medias pardas, tarsos amarillos, dos hileras de cerdas negras cortas en la parte inferior de los segmentos tarsales 1-3. Patas posteriores de color pardo obscuro, fémures más claros en los márgenes dorsal y ventral, tarsos amarillos. Último segmento tarsal infuscado en todas las patas. Gáster negro con brillo metálico. Vainas del ovipositor amarillas.

Estructura. Cabeza ligeramente más ancha que alta; frontovértice 0.3 veces la anchura de la cabeza. Ocelos en triángulo más o menos recto; ocelos posteriores separados del margen ocular por una distancia de un diámetro de un ocelo o por una distancia ligeramente más corta. Ojos 1.3 veces tan largos como las mejillas. Sutura genal completa. Área entre el margen interior del ojo y el margen escrobal casi tan ancha como en el margen inferior de los ojos (como en L. capitatus) pero 2 veces más angosta. Antenas insertadas ligeramente abajo del nivel del margen inferior de los ojos. Distancia entre tórulos más o menos como la distancia del tórulo al margen bucal y del tórulo al margen ocular. Radícula muy corta; escapo cilíndrico, ligeramente curvado, cerca de 6.0 veces tan largo como ancho. Anelo más o menos tan largo como ancho; segmentos funiculares I-III más largos que anchos y subiguales en longitud, segmento IV subcuadrado, segmentos V-VII ligeramente transversales. Maza antenal truncada oblicuamente y más corta que los tres segmentos funiculares precedentes juntos. Ala anterior 2.5 veces tan larga como ancha, vena marginal ligeramente más corta que la vena submarginal, venas postmarginal y estigmal subiguales en longitud, base de ala hialina. Fémur frontal ensanchado medialmente. Basitarso de la pata media ligeramente expandido y ligeramente más corto que los siguientes cuatro segmentos tarsales juntos. Segmentos tarsales 1-3 con dos hileras de espinas cortas. Tibia posterior más o menos tan larga como el fémur. Gáster más o menos tan largo como el tórax. Ovipositor ligeramente expuesto.

Longitud del cuerpo: $3.0 \mathrm{~mm}$.

Macho. Desconocido.

Distribución. México - Tamaulipas.

Huésped. Saissetia sp. 
Etimología. El nombre se deriva de México, por ser el país donde se colectó la nueva especie.

Comentarios. Esta nueva especie se distingue de las otras especies del género Lecaniobius en la cabeza, la cual es sólo ligeramente más ancha que larga y el frontovértice es 0.3 veces la anchura de la cabeza; área elevada entre las escrobas antenales y el margen de los ojos visiblemente más angosta; cuerpo completamente negro con brillo metálico; alas anteriores hialinas con una banda angosta infuscada debajo de la parte apical de la vena submarginal; tibias posteriores no más largas que los fémures posteriores.

\section{Lecaniobius nicaraguensis Myartseva, n. sp.}

Holotipo. \&, Nicaragua: Waspue, Riv. Musawas, 27-30.IX.1955 (col. B. Malkin); depositado en el Museo Essig, University of California-Berkeley.

\section{Descripción. Hembra.}

Coloración. Cabeza negra con brillo metálico verde en el occipucio y bronceado en las mejillas. Palpos labiales y maxilares amarillo claro. En el área elevada entre las escrobas, las setas plateadas son aplanadas, más grandes que las setas similares de las mejillas. Radícula y escapo parduzco, pedicelo y maza de pardo a negro, funículo pardo. Pronoto y mesotórax de color negro con brillo metálico, axilas negras, escutelo pardo y brillante, hilera media de cerdas ausente, pleuras con brillo metálico en la base. Alas anteriores con una banda obscura ancha que se extiende desde las venas marginal y estigmal hasta el borde posterior del ala y con una área obscura debajo de la vena submarginal que no alcanza el borde posterior del ala. Alas posteriores hialinas. Patas frontales pardas, coxas negras con brillo verde. Patas y coxas medias pardas, tarsos ligeramente más claros. Patas posteriores pardo obscuro, tibias y basitarso con margen dorsal blanco, segmentos tarsales III y IV amarillos. Gáster de parduzco a negro con brillo metálico, vainas expuestas del ovipositor amarillas con ápices negros.

Estructura. Cabeza más ancha que el tórax, 1.2 veces tan ancha como alta; frontovértice 0.4 veces la anchura de la cabeza. Ocelos dispuestos en un triángulo obtuso; ocelos posteriores separados del margen ocular por una distancia de cerca de 1.5 diámetros de un ocelo. Ojos 1.3 veces tan largos como las mejillas. Sutura genal completa. Área entre el margen interior del ojo y el margen escrobal casi tan ancha como en el margen inferior de los ojos. Antenas insertadas inmediatamente debajo del nivel del margen inferior de los ojos. Distancia entre tórulos ligeramente más larga que la distancia del tórulo al margen bucal y 0.5 veces tan larga como la distancia entre tórulos. Radícula muy corta; escapo cilíndrico, ligeramente curvado, cerca de 5.5 veces tan largo como ancho, anelo visiblemente más ancho que largo; segmentos funiculares I-III más largos que anchos y subiguales en longitud; segmentos IV y V ligeramente más anchos que largos; segmentos VI y VII subcuadrados. Maza antenal oblicuamente truncada, más o menos tan larga como los 
tres segmentos funiculares precedentes juntos. Escutelo sin hilera media de cerdas. Alas anteriores cerca de 2.2 veces tan largas como anchas; vena marginal visiblemente más corta que la vena submarginal; venas postmarginal y estigmal subiguales en longitud. Fémur frontal abultado distalmente. Basitarso de la pata media ligeramente expandido y ligeramente más corto que los siguientes cuatro segmentos tarsales juntos. Segmentos tarsales I-III con dos hileras de espinas cortas en el lado inferior. Tibia posterior algo comprimida y más larga que el fémur posterior. Gáster más o menos tan largo como el tórax, ovipositor expuesto.

Longitud del cuerpo: $3.9 \mathrm{~mm}$.

Macho. Desconocido.

Distribución. Nicaragua.

Huésped. Desconocido.

Etimología. El nombre se deriva de Nicaragua, el país de origen de esta nueva especie.

Comentarios. Esta nueva especie es similar a Lecaniobius cockerellii y a $L$. grandis por tener el margen blanco en las tibias posteriores pero difiere de ellas al presentar el margen dorsal blanco en el basitarso posterior. $L$. cockerellii tiene cabeza parda y cerca de 1.5 veces tan larga como ancha, ovipositor no expuesto mientras que L. nicaraguensis tiene cabeza de 1.2 veces tan ancha como larga y ovipositor expuesto. L. grandis presenta alas anteriores con un borde angosto contrastante de cilios blancos distal de la banda transversal ancha de cilios oscuros mientras que $L$. nicaraguensis no presenta dicho borde de cilios blancos en las alas anteriores.

\section{Lecaniobius utilis Compere}

Lecaniobius utilis Compere 1939: 79-82. Holotipo o, Brasil: Viscosa, Minas Geraes, 1933. USNM.

\section{Descripción. Hembra.}

Coloración. Cabeza y cuerpo obscuro, metálico, con reflejos fuertes azulados y violáceos, debajo de los ojos es pardo rojizo. Escapo antenal de color pardo rojizo, flagelo negruzco. Escutelo pardo obscuro. Patas frontales pardo obscuro. Tibia media con una mancha translúcida incolora en el ángulo apical aplanado. Base del gáster dorsalmente translúcido, parduzco o incoloro así como el ápice arqueado del terguito VII. Cabeza y mesoescudo con pubescencia reflejante blanco plateado. En la área elevada entre las escrobas, las setas plateadas son aplanadas, más largas que las setas similares en las mejillas. Escutelo con una hilera longitudinal media de setas burdas negras y cortas. Ala anterior con una banda obscura ancha que cruza por en medio el disco, mitad apical del ala ligeramente ahumada, base con área infuscada debajo de la parte basal de la vena submarginal que no alcanza el borde posterior del ala.

Estructura. Cabeza cerca de 1.4 veces tan ancha como alta. Margen occipital redondeado. Frontovértice cerca de 0.5 veces la anchura de la cabeza. Ojos 1.3 veces 
tan largos como las mejillas. Área elevada entre las escrobas con setas plateadas aplanadas, más largas que las similares en las mejillas. Antenas insertadas inmediatamente al nivel del margen inferior de los ojos. Escapo 5.6 veces tan largo como ancho, pedicelo cerca de 2.0 veces tan largo como ancho, anelo ligeramente más ancho que largo. Segmento funicular I cerca de dos veces tan largo como ancho, segmentos II y III cerca de 1.5 veces tan largos como anchos, segmento IV ligeramente más largo que ancho, segmentos V-VII ligeramente más anchos que largos. Maza antenal truncada oblicuamente, ligeramente más corta que los tres segmentos funiculares precedentes juntos. Todos los segmentos flagelares, excepto el primero, con sensilas longitudinales. Ala anterior 2.2 veces tan larga como ancha, vena marginal ligeramente más corta que la vena submarginal, venas postmarginal y estigmal subiguales en longitud. Fémur frontal abultado. Basitarso medio ligeramente ensanchado. Gáster más o menos tan largo como el tórax. Ovipositor ligeramente expuesto.

Longitud del cuerpo: $3.0 \mathrm{~mm}$.

Macho. Similar en coloración y estructura a la hembra excepto por la genitalia y las antenas. Todos los tarsos principalmente blancos, segmento apical oscuro; espuela blanca en la tibia media; espuelas apareadas de las tibias posteriores obscuras; margen apical de la tibia media con una traza blancuzca. Cabeza casi tan alta como ancha; pedicelo pequeño, ligeramente más largo que ancho; segmentos funiculares ligeramente disminuyendo en longitud, los primeros cuatro segmentos visiblemente más largos que anchos; todos los segmentos flagelares con muchas sensilas.

Longitud del cuerpo: $2.0 \mathrm{~mm}$.

Comentarios. Lecaniobius utilis es una especie cercana a $L$. capitatus, se distinguen en las siguientes características: L. capitatus - frontovértice 0.4 veces la anchura de la cabeza, ala anterior tres veces tan larga como ancha y su base con una banda oblicua ahumada y una banda oscura media marginada por un borde angosto contrastante de cilios blancos distales; $L$. utilis - frontovértice 0.5 veces la anchura de la cabeza, ala anterior 2.2 veces tan larga como ancha y su base con una área grande infuscada debajo de la vena submarginal, la banda obscura media sin un borde angosto contrastante de cilios blancos distales.

Lecaniobius utilis fue introducida desde Brasil y Argentina a California, EU, en 1934-1935 y se estableció (Flanders 1942). Después fue introducida desde California a Perú y fue la especie más eficiente (en comparación con las otras introducidas) ya que se obtenía cerca del $90 \%$ de parasitismo en campo en Saissetia oleae (Beingolea 1956). Esta especie ha sido utilizada para el control biológico de la escama negra en Norte y Sudamérica (Gibson 1995b).

Distribución. Argentina, Brasil, Chile, Paraguay, Perú, Uruguay, EU (California) (Noyes 2003). 
Huéspedes. Parasaissetia nigra (Nietner), Saissetia coffeae (Walker), S. discoides (Hempel) y $S$. oleae (Olivier).

Material examinado. Paratipos $34 \uparrow$, Riverside, California, Insectary (parent stock coll., Brazil 1934), H. Compere - en el Museo Essig, University of CaliforniaBerkeley.

AGRADECIMIENTOS. A la Dra. Cheryl B. Barr (Essig Museum of Entomology, University of California, Berkeley, EU) por el préstamo de materiales de Eupelmidae para su estudio, al Dr. W.J. Pulawski por su ayuda en la búsqueda de materiales en el Entomological Museum de la California Academy of Sciences, San Francisco, EU. Al proyecto PROMEP "Taxonomía y ecología de fauna y micobiota en comunidades forestales y cultivos de México" de la Red de Cuerpos Académicos PROMEP, y a la UAT por su apoyo para la realización de este trabajo.

\section{LITERATURA CITADA}

Ashmead, W.H. 1896. On the genera of the Eupelminae. Proceedings of the Entomological Society of Washington, 4 (1): 4-20.

Bartlett, B.R. 1978. Coccidae, pp. 57-74. En: Clausen, C.P. (Ed.). Introduced parasites and predators of arthropod pests and weeds: A world review. U.S. Dept. Agric. Handbook, 480. 545 pp.

Beingolea, G.O. 1956. Estatus actual de la plaga de la quereza negra del olivo (Saissetia oleae Bern.) en los valles de Yauca E Ilo. Bol. Trimest. Expt. Agropecuarias, 4: 18-22.

Blanchard, E.E. 1956. A new eupelmid parasitising a coccid. Anales de la Sociedad Científica Argentina, 162 (5-6): 160-164.

Burks, B.D. 1979. Torymidae (Agaoninae) and all other families of Chalcidoidea (excluding Encyrtidae). En: Krombein, K.V., Hurd, P.D. jr., Smith, D.R. \& Burks, B.D. (Eds.). Catalog of Hymenoptera in America North of Mexico, 1. Smithsonian Institute Press, Washington, D.C. 889 pp.

Compere, H. 1939. The insect enemies of the black scale, Saissetia oleae (Bern.) in South America. University of California Publications in Entomology, 7: 75-90.

Crawford, J.C. 1908. A new genus and species of Eupelminae. Proceedings of the Entomological Society of Washington, 9: 156-157.

Crawford, J.C. 1911. Descriptions of new Hymenoptera. 3. Proceedings of the United States National Museum, 41: 267-282.

De Santis, L. 1950. Descripción de un nuevo eupélmido de la República Argentina (Hymenoptera: Chalcidoidea). Notas del Museo de La Plata, 15 (127): 1-5.

De Santis, L. 1979. Catálogo de los himenópteros calcidoideos al sur de los Estados Unidos. Publicación Especial, La Plata, República Argentina. 488 pp.

De Santis, L. y A.E. Gallego de Sureda. 1986. Nota sinonímica sobre un eupélmido (Hym.) parasitoide de la cochinilla del aguaribay (Hom. Coccidae). Revista de la Asociación de Ciencias Naturales del Litoral, 17: 207-209.

Essig, E.O. 1931. A History of Entomology. The Macmillan Company. New York. 1029 pp.

Flanders, S.E. 1942. Propagation on black scale on potato sprouts. Journal of Economic Entomology, 35 (5): 687-689.

Flanders, S.E. 1953. Variations in susceptibility of Citrus-infesting coccids to parasitization. Journal of Economic Entomology, 46 (2): 266-269.

González-Hernández, A. 2000. Chalcidoidea, pp. 649-659. En: Bousquets, J.L., E. González Soriano, \& N. Papavero (Eds.). Biodiversidad, taxonomía y biogeografía de artrópodos de México: Hacia una síntesis de su conocimiento. Vol. II. UNAM. México. 
Gahan, A.B. 1924. Some new parasitic Hymenoptera with notes in several described forms. Proceedings of U.S. Natural Museum, 65: 1-23.

Gibson, G.A.P. 1995a. Parasitic wasps of the subfamily Eupelminae: classification and revision of world genera (Hym.: Chalc.: Eupelm.). Memoirs on Entomology, International, 5. Associated Publishers, Ottawa, Canada. 421 pp.

Gibson, G.A.P. 1995b. Eupelmidae, pp. 329-336. En: Hanson, P.E. \& I.D. Gauld (Eds.). The Hymenoptera of Costa Rica. Oxford University Press. UK. 893 pp.

Griot, M. 1955. Observaciones sobre algunos parásitos de Ceroplastes grandis Hempel. Revista de la Facultad de Agronomía y Veterinaria, Universidad de Buenos Aires, 13: 491-504.

Myartseva, S.N., E. Ruíz-Cancino \& J.M. Coronado-Blanco. 2004. Parasitoids (Hymenoptera: Chalcidoidea) of Saissetia spp. (Homoptera: Coccidae) in Mexico. Fruits, 59: 141-150.

Noyes, J.S. 1982. Collecting and preserving chalcid wasps (Hymenoptera:Chalcidoidea). Journal of Natural History, 16: 315-334.

Noyes, J.S. 2003. Universal Chalcidoidea Database [online]. www.nhm.ac.uk/entomology/chalcidoids/index.html. (Database last updated: April 2009).

Ruíz Cancino, E. \& J.M. Coronado Blanco. 2002. Artrópodos terrestres de los estados de Tamaulipas y Nuevo León, México. Serie Publicaciones Científicas CIDAFF-UAT No. 3. Cd. Victoria, Tamaulipas, México. 377 pp.

Smith, H.S. 1923. Biological control work. The Monthly Bulletin of California Department of Agriculture, 12 (7-12): 334-339.

Smith, R.H. 1944. Bionomics and control of the nigra scale, Saissetia nigra. Hilgardia, 16 (5): 225288.

Smith, H.S. \& H. Compere. 1928. A preliminary report on the insect parasites of the black scale, Saissetia oleae (Bern.). University of California Publications in Entomology, 4: 232-334. 